\title{
Synthesis and Molecular Characterization of A-B-A Tri-Block Copolymers Composed of Poly ( $\gamma$-ethyl L-glutamate) as the A Component and Polybutadiene as the B Component
}

\author{
Guan-Wen Chen, * Toshio Hayashi, and Akio NAKAJIMA
}

\author{
Department of Polymer Chemistry, Kyoto University, \\ Yoshida, Sakyo-ku, Kyoto 606, Japan.
}

(Received July 18, 1980)

\begin{abstract}
A series of A-B-A tri-block copolymers consisting of poly( $\gamma$-ethyl L-glutamate) as outer blocks and polybutadiene as the middle block were synthesized by polymerizing $\gamma$-ethyl Lglutamate $N$-carboxy anhydride at the ends of the amine-terminated polybutadiene, in which the terminal amine groups act as the initiator. In the preparation of $\gamma$-ethyl L-glutamate from Lglutamic acid, an effective method to minimize the formation of diester was found. The molecular weights and molecular conformation of the polymers in helicogenic solvent were determined by elemental analysis, ultracentrifugation, optical rotatory dispersion, and circular dichroism. It was found that the A block portion behaves as the $\alpha$-helix and the block copolymer chain can be treated as a once-broken rod. Model analysis of the complex modulus of the membrane cast from solution suggested the occurrence of spherical and cylindrical domain structures in the membrane.

KEY WORDS A-B-A Type Tri-Block Copolymer/Polybutadiene / Poly $(\gamma$ ethyl L-glutamate) / Synthesis /Characterization / Complex Modulus / Chain Conformation /
\end{abstract}

Block copolymers of the A-B di-block and A-B-A tri-block types are of special interest from the standpoint of molecular designing. These copolymers are capable of forming organized structures, i.e., microheterophase structures, when cast from solutions, and the resulting membranes are expected to exhibit specific functionalities. Therefore, it is not surprising that a number of papers have been published on this subject. However, most of the previous papers concerned themselves with block copolymers in which both the $\mathrm{A}$ and $\mathrm{B}$ component blocks are flexible chains so that the polymers assume random coil conformations in solution.

Attempts to incorporate a polypeptide as one of the components of $\mathrm{A}-\mathrm{B}$ and $\mathrm{A}-\mathrm{B}-\mathrm{A}$ type block copolymers have been made by several authors. ${ }^{1-3}$ Douy and Gallot ${ }^{2}$ first reported the organized struc-

* On leave from Institute of Chemistry, Chinese Academy of Sciences, Peking, The People's Republic of China. ture which occurred with an A-B di-block copolymer containing a polypeptide as the A component. We have recently advanced a series of works ${ }^{4-7}$ on the organized structure of A-B-A triblock copolymers, using $\gamma$-benzyl L-glutamate, $\gamma$ methyl L-glutamate, and $N$-benzyloxycarbonyl Llysine as the A component, and butadiene and tetramethylene oxide as the B components.

In this paper, we report on the synthesis and molecular characterization of a series of A-B-A triblock copolymers consisting of $\gamma$-ethyl L-glutamate as the $\mathrm{A}$ component and butadiene as the $\mathrm{B}$ component.

Poly( $\gamma$-ethyl L-glutamate) (abbreviated as PELG) is similar to poly $(\gamma$-methyl L-glutamate) (abbr. as PMLG) in molecular structure, but its helix stability and solubility in polar solvent are less than those of PMLG. Thus, the water vapor absorption of PELG is less than that of PMLG. However, the reverse was found ${ }^{8}$ for water permeability through the membrane. For such behavior Takizawa ${ }^{8} \mathrm{em}$ - 
phasized the importance of the diffusion of water in the PELG membrane. One purpose for investigating the A-B-A block copolymer composed of PELG and polybutadiene is the application of this material in biomedical use. Hydrolysis of PELG in a living body may produce ethyl alcohol, which is less injurious than methyl alcohol, a hydrolysis product of PMLG, as was suggested by Nose. ${ }^{9}$

\section{EXPERIMENTAL}

\section{Synthesis of Samples}

Amine-Terminated Polybutadiene. The middle block, a cycloaliphatic secondary amine-terminated polybutadiene (ATPB), in which the content of trans isomer is high, was kindly supplied by Doctors K. Rieu and R. Drake of the B. F. Goodrich Chem. Co., Cleveland, Ohio. They prepared the ATPB in the following way. A carboxylated polybutadiene (Hycar CTB) was transformed into di-aminated polybutadiene (ATPB) by reacting $N$-aminoethylpiperadine. The extent of amination was measured by potentiometric titration. The ratio of the weightaverage molecular weight $M_{w}$ to the numberaverage molecular weight $M_{n}$ of the ATPB was estimated to be 1.05 by gel-permeation chromatography. The ATPB was purified before use by using benzene, methanol, and water. The amine equivalent weight of the ATPB was measured to be 1800 by potentiometric titration. Thus, the numberaverage molecular weight $M_{n}$ of the ATPB was 3600. The molecular formula of the ATPB is
$\gamma$-Ethyl L-Glutamate. The monomer $\gamma$-ethyl Lglutamate was prepared by the method originally proposed by Pravda ${ }^{10}$ and modified by us. First, a saturated solution of $50 \mathrm{~g} \mathrm{~L}$-glutamic acid (Nakarai Chem. Co., Reagent Grade) in $500 \mathrm{ml}$ of water at an elevated temperature around $90^{\circ} \mathrm{C}$ was cooled to $20^{\circ} \mathrm{C}$ under vigorous stirring in $5 \mathrm{~min}$ by means of a freezing mixture. At the same time, an equal volume of ethanol $(500 \mathrm{ml})$ was added to the solution. The crystall of L-glutamic acid formed were collected on a No. 4 glass filter and then dried in vacuo. This pretreated L-glutamic acid was then used for esterification. A suspension of $35 \mathrm{~g}(0.24 \mathrm{~mol}) \mathrm{L}$-glutamic acid in $350 \mathrm{ml}$ absolute ethanol containing about $25 \mathrm{~g}(0.68 \mathrm{~mol})$ dry hydrogen chloride was shaken for $10 \mathrm{~min}$ at $20^{\circ} \mathrm{C}$. This operation should be completed within $10 \mathrm{~min}$ and the reaction temperature should be kept at a temperature not exceeding $20^{\circ} \mathrm{C}$, to minimize formation of the diester. By this reaction, most of the solid was dissolved. After removing the remaining traces by filtration, the clear solution was diluted with $500 \mathrm{ml}$ of absolute ethanol, and cooled to $0-5^{\circ} \mathrm{C}$. Triethylamine (TEA) was added dropwise at a temperature not higher than $5^{\circ} \mathrm{C}$ until the $\mathrm{pH}$ of the solution became about 6.5-7.0. After standing for $12 \mathrm{~h}$ at $0-5^{\circ} \mathrm{C}$, the product was filtered, washed several times with cold absolute ethanol and ether, and air-dried on the filter glass. Drying was completed in vacuo with an yield of $29 \mathrm{~g}(70 \%)$. The product was further recrystallized from an ethanol-water system. The melting point of the final $\gamma$-ethyl L-glutamate was

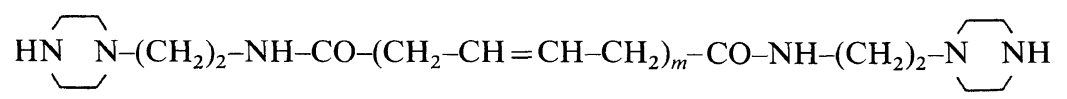

$188-190^{\circ} \mathrm{C}$, which can be compared with the literature values of $\mathrm{Sugai}^{11}\left(186-190^{\circ} \mathrm{C}\right)$ and of Greenstein $^{12}\left(194^{\circ} \mathrm{C}\right)$. By thin layer chromatography with the pyridine-methanol-chloroform system, it was confirmed that the product scarcely contained $\gamma, \alpha$-diethyl ester. Elemental analysis yielded: C, $47.49 \%, 7.25 \%$, and $\mathrm{N}, 8.08 \%$, and these values are comparable to the calculated values for $\gamma$-ethyl L-glutamate: $\mathrm{C}, 47.99 \%, \mathrm{H}, 7.48 \%$, and $\mathrm{N}$, $8.00 \%$.

$\gamma$-Eethyl L-Glutamate $N$-Carboxy Anhydride. $\gamma$ Ethyl L-glutamate NCA ( $\gamma$-ELG-NCA), the monomer for the outer block, was prepared according to the method of Stewart and Ledger, ${ }^{13}$ and purified by repeated recrystallization from an ethyl acetate solution with the addition of petroleum ether.

Synthesis of Block Copolymers. Appropriate amounts of $\gamma$-ELG-NCA and ATPB were calculated according to $P_{n}=[\mathrm{NCA}] /[\mathrm{ATPB}]$ to obtain the desired degree of polymerization, $P_{n}$, of the polypeptide block, in which [NCA] and [ATPB] are the molar concentrations of NCA and ATPB, respectively. The polymerization was carried out in the absence of moisture at $20^{\circ} \mathrm{C}$ in a dioxane-methylene dichloride $(1: 2 \mathrm{v} / \mathrm{v})$ mixture at a total concentration of $\gamma$-ELG-NCA and ATPB, 3\%. After $72 \mathrm{~h}$, the polymerization was terminated, and the copolymer was precipitated with 4 volumes of pure cold 
ethanol. This method of precipitation allowed to elimination of traces of $\gamma$-ELG-NCA still present and the oligopeptide formed by autopolymerization.

\section{Measurements}

Composition of Block Copolymers. The composition of $\gamma$-ELG in each sample of block copolymer was determined by elemental analyses and infrared (IR) spectra. The elemental analyses were carried out in the Organic Microanalyses Center in Kyoto University, and the IR spectra of the solid films of the samples cast from a chloroform-trifluoroethanol mixture were measured with a Shimadzu Model-30 A infrared spectrophotometer in a region of 4000 to $400 \mathrm{~cm}^{-1}$.

Since the molecular weight of the middle block is known, the molecular weight of the outer block, a polypeptide block, can be estimated from the copolymer composition. The molecular weight thus obtained is the number-average molecular weight.

Molecular Weight Determination by Ultracentrifugation. The molecular weight of the block copolymer EBE-10 was determined in $m$-cresol at $25^{\circ} \mathrm{C}$ by sedimentation equilibrium using a MOM Type-3170-b ultracentrifuge equipped with the Reyleigh interference optical system and a $12 \mathrm{~mm}$ double sector cell. Concentration differences between the solvent in one sector and the sample + solvent in the other were determined at various points in the cell by counting fringes. The ultracentrifuge was operated at a rotor speed of $8000 \mathrm{rpm}$ for $48 \mathrm{~h}$ to attain the equilibrium. The light source was a. high-pressure mercury lamp with an interference filter to isolate the $546 \mathrm{~nm}$ line. Sedimentation patterns were photographed on Fuji panchromatic films. The patterns were read by using a Nikon profile projector V-16 (Nihon Kogaku, Tokyo) to an accuracy of $\pm 0.05 \mathrm{~mm}$, which corresponds to about $0.0025 \mathrm{~mm}$ in the cell.

The weight-average molecular weight $M_{w r}$ at a radial distance $r$ from the center of rotation is given by,

$$
M_{w r}=\frac{2 R T}{\omega^{2}(1-\bar{v} \rho)} \cdot \frac{\mathrm{d} \ln c}{\mathrm{~d} r^{2}}
$$

where $R$ is the universal gas constant, $T$ is the absolute temperature, $\omega$ is the angular velocity of the rotor, $\bar{v}$ is the partial specific volume of the solute $\left(\bar{v}=0.7692 \mathrm{~cm}^{3} \mathrm{~g}^{-1}\right), \rho$ is the density of the solution $\left(\rho=1.034 \mathrm{~g} \mathrm{~cm}^{-3}\right)$, and $c$ is the concen- tration of the polymer. For a polydisperse system, $M_{w r}$ takes different values at different points $r$ in the cell. The apparent weight-average molecular weight $M_{w}^{\text {app }}$ of the whole sample in the cell is obtained from eq 2 as a function of the polymer concentration $c_{0}$ before centrifugation,

$$
M_{w}^{\mathrm{app}}=\frac{2 R T\left(c_{\mathrm{b}}-c_{\mathrm{m}}\right)}{\omega^{2}(1-\bar{v} \rho) c_{0}\left(r_{\mathrm{b}}{ }^{2}-r_{\mathrm{m}}{ }^{2}\right)}
$$

where $c_{\mathrm{b}}$ and $c_{\mathrm{m}}$ are the equilibrium concentrations at the bottom of the cell $r_{\mathrm{b}}$ and at the meniscus $r_{\mathrm{m}}$, respectively. Under specified centrifugation conditions the solute distribution is dependent not only on molecular weight of the solute but also on thermodynamic quantities such as specific volume and thermodynamic nonideality. Further, the optical system installed in the ultracentrifuge records only the solute distribution in terms of certain optical quantities. Thus, when a nonideal solution of a heterogeneous solute, such as block copolymer EBE-10, is to be examined, we should take account of the heterogeneities not only in molecular weight but also in partial specific volume, thermodynamic interaction parameter, and specific refractive increment. ${ }^{14}$ However, we simply used eq 2 for determining the apparent weight-average molecular weight, $M_{w}^{\text {app }}$, assuming that the small amount of the polybutadiene portion would not measurably affect the thermodynamic nonideality. The results obtained are $M_{w}^{\text {app }}=12.5 \times 10^{4}$ at $c_{0}=0.074 \mathrm{~g} \mathrm{dl}^{-1}, 12.4 \times 10^{4}$ at $c_{0}=0.11 \mathrm{~g} \mathrm{dl}^{-1}, 12.4 \times 10^{4}$ at $c_{0}=0.16 \mathrm{~g} \mathrm{dl}^{-1}$, and $12.2 \times 10^{4}$ at $c_{0}=0.32 \mathrm{~g} \mathrm{dl}^{-1}$. By extrapolating to infinite dilution, we obtain $12.5 \times 10^{4}$ for the weightaverage molecular weight $M_{w}$. Since the $M_{w}^{\text {app }}$ values of the EBE-10 sample were found to be almost independent of concentration over the range from 0.074 to $0.32\left(\mathrm{~g} \mathrm{dl}^{-1}\right)$, we show only the data for the lowest concentration in Table II.

The $z$-average molecular weight of the whole sample, $M_{z}^{\mathrm{app}}$, is given by,

$$
M_{z}^{\mathrm{app}}=\frac{M_{w \mathrm{~b}} c_{\mathrm{b}}-M_{w \mathrm{~m}} c_{\mathrm{m}}}{c_{\mathrm{b}}-c_{\mathrm{m}}}
$$

The numerical values of $M_{w \mathrm{~b}}, M_{w \mathrm{~m}}$, and $M_{z}$, obtained for the lowest concentration $\left(c_{0}=0.074\right.$ $\mathrm{g} \mathrm{dl}^{-1}$ ) of EBE-10 are also shown in Table II. In addition to $M_{w}$ and $M_{z}$, the number-average molecular weight $M_{n}$ for EBE-10 was estimated also at $c_{0}=0.074 \mathrm{~g} \mathrm{dl}^{-1}$ using the meniscus depletion meth$\mathrm{od}^{15}$ and the rotor speed of $18000 \mathrm{rpm}$. 
Chain Conformation of PELG Portion of the Block Copolymer. The chain conformation of the PELG portion of the block copolymer in trifluoroethanol was examined by circular dichroism (CD) specta. The measurements were made at $25^{\circ} \mathrm{C}$ by a JASCO J-20 CD/ORD spectropolarimeter. The helical content $X_{\mathrm{H}}$ is defined by the ratio $[\theta]_{222}^{c} /$ $[\theta]_{222}^{0}$, where $[\theta]_{222}^{c}$ is the residue ellipticity at $222 \mathrm{~nm}$ of the block copolymer, and $[\theta]_{222}^{0}$ is that of PELG homopolymer. The Moffitt parameter $b_{0}$ was evaluated from the optical rotatory dispersion (ORD) data measured by a Yanagimoto OR-100 Type spectropolarimeter, using a tungsten lamp as the light source in a temperature range of $10-40^{\circ} \mathrm{C}$. The reduced residue rotation $\left[\mathrm{m}^{\prime}\right]_{\lambda}$ at a wave length $\lambda$ is obtained from the observed rotation $\alpha_{\lambda}$ by

$$
\left[m^{\prime}\right]_{\lambda}=\left[3 /\left(n^{2}+2\right)\right](100 / c l) \alpha_{\lambda}
$$

where $n$ is the refractive index of the solution, $c$ the polymer concentration in mol residue per liter, and $l$ is the cell path length in $\mathrm{cm}$. The Moffitt parameter $b_{0}$ was obtained from the slope of the plot of $\left[m^{\prime}\right]_{\lambda}\left(\lambda^{2}-\lambda_{0}^{2}\right) / \lambda_{0}{ }^{2}$ against $\lambda_{0}^{2} /\left(\lambda^{2}-\lambda_{0}{ }^{2}\right)$, with 212 $\mathrm{nm}$ assumed for $\lambda_{0}$.

The chain conformation of the PELG portion in the solid film cast from trifluoroethanol solution was estimated from IR spectra measured by a Shimadzu Model-30A IR spectrophotometer.

Dynamic Mechanical Properties. The loss tangent $\tan \delta$ and the storage modulus $E^{\prime}$ were measured with a DDV-II Rheovibron at a frequency of $110 \mathrm{~Hz}$ and a heating rate of $0.3^{\circ} \mathrm{C} \mathrm{min}{ }^{-1}$ over a temperature range from -20 to $100^{\circ} \mathrm{C}$. The samples used were the non-oriented solid films, ca. $50 \mu \mathrm{m}$ in thickness, of the block copolymers and the PELG homopolymers.

\section{RESULTS AND DISCUSSION}

Molecular Weights and Molecular Compositions of EBE Block Copolymers, and Their Molecular Conformations in Solution

A series of A-B-A triblock copolymers consisting of PELG as the A blocks and polybutadiene as the B block are hereafter designated as EBE copolymers. The compositions, degree of polymerization, $P_{\mathrm{A}}$, of the A block chain, and the helical content $X_{\mathrm{H}}$ of EBE copolymers are shown in Table $\mathrm{I}$, in which the $P_{\mathrm{A}}$ values of the EBE copolymers are those estimated from the copolymer composition. Table I also includes the number-average degrees of polymerization of PELG homopolymers estimated from the limiting viscosity numbers $[\eta]$ in dichloroacetic acid at $25^{\circ} \mathrm{C}$ by using the [ $\left.\eta\right] v s$. molecular weight $M_{n}$ relationship proposed ${ }^{16}$ for PMLG.

One of the EBE copolymers, EBE-10, was characterized by ultracentrifugation. The weightaverage molecular weight $M_{w}$ was obtained from eq 1. Figure 1 shows the plot of $\ln c$ against $r^{2}$ for EBE-10 in $m$-cresol at sedimentation equilibrium in $48 \mathrm{~h}$ at $8000 \mathrm{rpm}$ from which $M_{w \mathrm{~m}}$ and $M_{w \mathrm{~b}}$ were estimated by eq 3 and the $z$-average molecular weight $M_{z}$ was determined from eq 2. Figure 2 is the plot of $c$ against $r^{2}$ at sedimentation equilibrium in $12 \mathrm{~h}$ at a higher speed of rotation $18,000 \mathrm{rpm}$. The numberaverage molecular weight $M_{n}$ was estimated from the plot using the meniscus depletion method.

Table II summarizes the results from ultracentrifugal measurements together with the value of

Table I. Molecular characterization of samples

\begin{tabular}{cccc}
\hline & $\gamma$-ELG & & \\
\cline { 2 - 2 } Sample code & & $P_{\mathrm{A}}$ & $x_{\mathrm{H}}$ \\
& mol $\%$ & & \\
\hline EBE-05 & 94.5 & 495 & 0.95 \\
EBE-10 & 89.5 & 260 & 0.89 \\
EBE-20 & 80.6 & 127 & 0.80 \\
EBE-30 & 69.1 & 68 & 0.68 \\
EBE-40 & 60.5 & 47 & 0.60 \\
& & & \\
PELG-1 & 100 & $4050^{\mathrm{a}}$ & 1 \\
PELG-2 & 100 & $588^{\mathrm{a}}$ & 1 \\
PELG-3 & 100 & $249^{\mathrm{a}}$ & 1 \\
\hline
\end{tabular}

a Degree of polymerization of whole polymer $\left(=2 P_{\mathrm{A}}\right)$.

Table II. Average molecular weights of EBE-10

\begin{tabular}{lcccccc}
\hline & $M_{w}$ & $M_{w \mathrm{~m}}$ & $M_{w \mathrm{~b}}$ & $M_{z}$ & $M_{z} / M_{w}$ & $M_{n}$ \\
\hline $\begin{array}{l}\text { Exptl } \\
\text { Calcd }\end{array}$ & $12.5 \times \mathrm{i} 0^{4}$ & $11.0 \times 10^{4}$ & $15.0 \times 10^{4}$ & $15.8 \times 10^{4}$ & 1.26 & $\begin{array}{l}9.0 \times 10^{4} \\
9.26 \times 10^{4}\end{array}$ \\
\hline
\end{tabular}




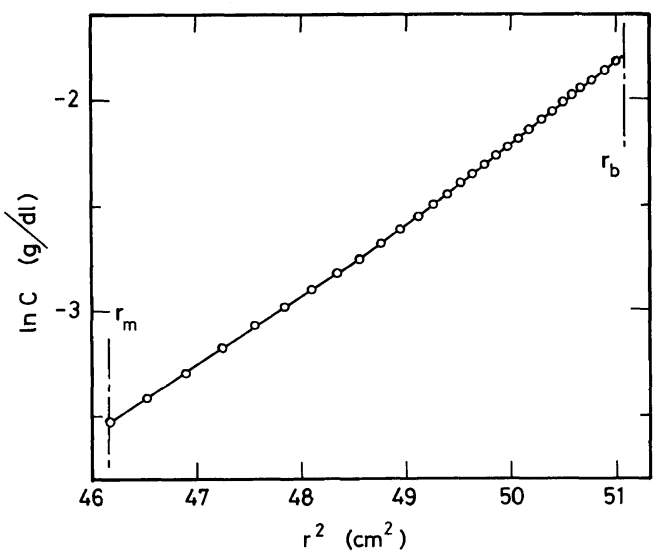

Figure 1. Plot of $\ln c$ against $r^{2}$ for EBE-10 in $m$-cresol at sedimentation equilibrium at $8000 \mathrm{rpm}$.

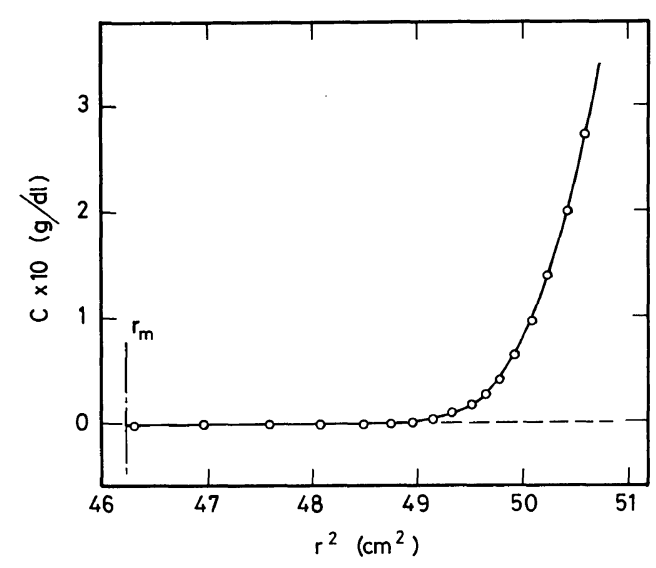

Figure 2. Plot of $c$ against $r^{2}$ for EBE-10 in $m$-cresol at sedimentation equilibrium at $18000 \mathrm{rpm}$ and $12 \mathrm{~h}$.

$M_{n}$ calculated from $M_{z} / M_{w}=1.26$ by assuming the generalized exponential distribution function, in which the relation $M_{n}: M_{w}: M_{z}=(b+1):(b+2)$ : $(b+3)$ holds. We note that the $M_{n}$ value $\left(9.0 \times 10^{4}\right)$ directly obtained from the centrifugal measurement is in good agreement with that $\left(9.26 \times 10^{4}\right)$ calculated from the $M_{z} / M_{w}$ values.

Further, these $M_{n}$ values compare well with the $M_{n}$, value estimated from the copolymer composition; $P_{\mathrm{A}}=260$ (see EBE-10 in Table I) leads to $M_{n}=8.5 \times 10^{4}$ for EBE-10 copolymer. This agreement-supports the conclusion that our block copolymer is of A-B-A tri-block type, and consists of $A$ block chains of equal length linked to the ends of the $\mathrm{B}$ chain, produced by nucleophilic addi-

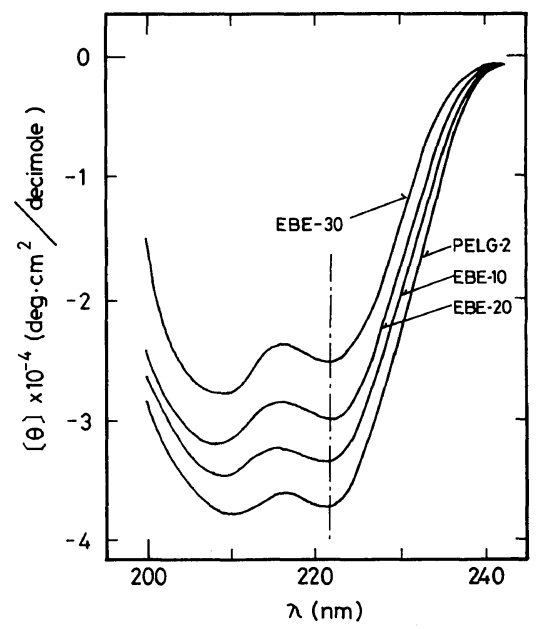

Figure 3. Circular dichroism spectra of EBE block copolymers and PELG homopolymer in trifluoroethanol at $25^{\circ} \mathrm{C}$.

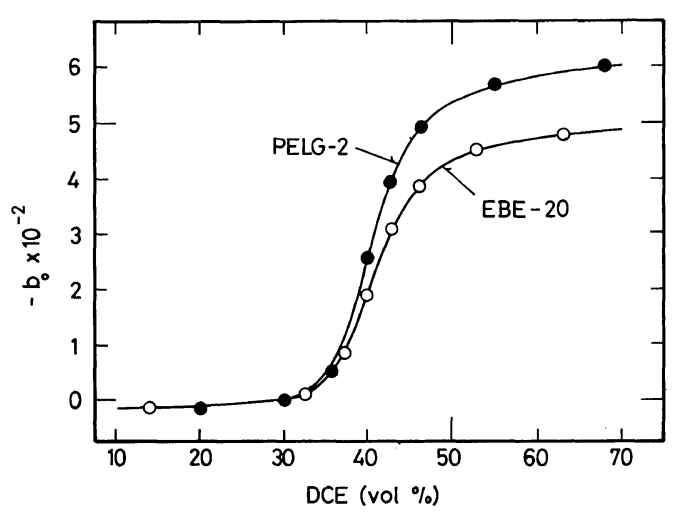

Figure 4. Helix-coil transition of EBE-20 and PELG-2 in DCA-DCE mixtures at $25^{\circ} \mathrm{C}$.

tion $^{17}$ of $\gamma$-ELG-NCA unique the cyclic secondary amines. And, located at the ends of the B chain.

From these results, the molecular weight heterogeneity of the block copolymer is represented by $M_{w} / M_{n}=1.4$ and $M_{z} / M_{w}=1.25$.

Table I also presents information on the molecular conformation of EBE copolymers. The helical contents $X_{\mathrm{H}}$ of the EBE copolymers were determined in trifluoroethanol, a helicogenic solvent. We see that $X_{\mathrm{H}}$ for each of the EBE block copolymers is in close agreement with the molar composition of the $\gamma$-ELG blocks in the copolymers. This indicates that the helical content of the $\gamma$-ELG component in 
EBE copolymers is equal to that of the PELG homopolymer in the helicogenic solvent. In Figure 3, CD spectra of EBE copolymers and PELG homopolymer are shown. The $X_{\mathrm{H}}$ values given in Table I were obtained from the $[\theta]$ values at $222 \mathrm{~nm}$.

The helix stability was investigated by observing the helix-coil transition as a function of solvent composition and temperature. Figure 4 shows the helix-coil transitions of EBE copolymer and PELG homopolymer in mixtures of dichloroacetic acid (DCA) and dichloroethane (DCE) at $25^{\circ} \mathrm{C}$. The midpoint of the helix-coil transition is located at about a $40 \%$ DCE content for both EBE-20 and PELG-2. Further, the $b_{0}$ value of EBE-20 at high DCE content is about $80 \%$ of that of PELG-2, which is consistent with the value expected from the copolymer composition. Taking into consideration that the midpoint of the helix-coil transition of PMLG in DCA-DCE system appears at about $30 \%$ DCE content, ${ }^{18}$ we suggest that the helix stability of PELG is less than that of PMLG.

Next we deal with the thermal helix-coil transition of EBE copolymers in a 43:57 DCA-DCE mixture. In Figure 5, the Moffitt parameter $b_{0}$ is plotted against temperature for EBE copolymers and for PELG homopolymers of different molecular weights. As is obvious from the figure, the transition temperature $T_{\mathrm{t}}$ gets higher as the molecular weight of PELG is decreased.

According to Lifson and Roig ${ }^{19}$ and Nagai, ${ }^{20} T_{\mathrm{t}}$ of a homopolypeptide is given by a series of the reciprocal number-average molecular weight, $M_{n}^{-1}$,

$$
T_{\mathrm{t}}=T_{\mathrm{t}}^{\infty}+K^{\prime} / M_{n}+0\left(1 / M_{n}^{2}\right)
$$

where $T_{\mathrm{t}}{ }^{\infty}$ is the $T_{\mathrm{t}}$ for infinite molecular weight and $K^{\prime}$ is a constant independent of $M_{n}$. By extending the above equation to the case in which the polypeptide molecule contains one intrachain defect which cannot participate in the formation of helix. Teramoto et al. ${ }^{21}$ proposed the following equation:

$$
T_{\mathrm{t}}=T_{\mathrm{t}}^{\infty}+2 \beta K^{\prime} / M_{n}+0\left(1 / M_{n}{ }^{2}\right)
$$

where $\beta$ is a parameter associated with the chemical nature of the defect, and takes a value of unity if the defect acts in the same way as the chain end of the molecule does in the formation of helix.

Now, our A-B-A tri-block copolymer can be regarded as a once-broken rod which may be treated by eq 6 with $\beta=1$. In Figure $6, T_{\mathrm{t}}$ obtained from Figure 5 are plotted against the reciprocal number-

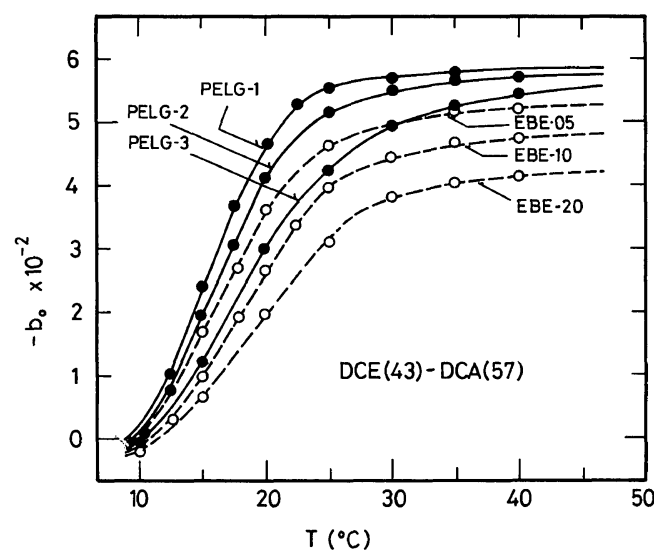

Figure 5. Helix-coil transition of EBE copolymers and PELG homopolymers in 43:57 DCE-DCA mixture as function of temperature.

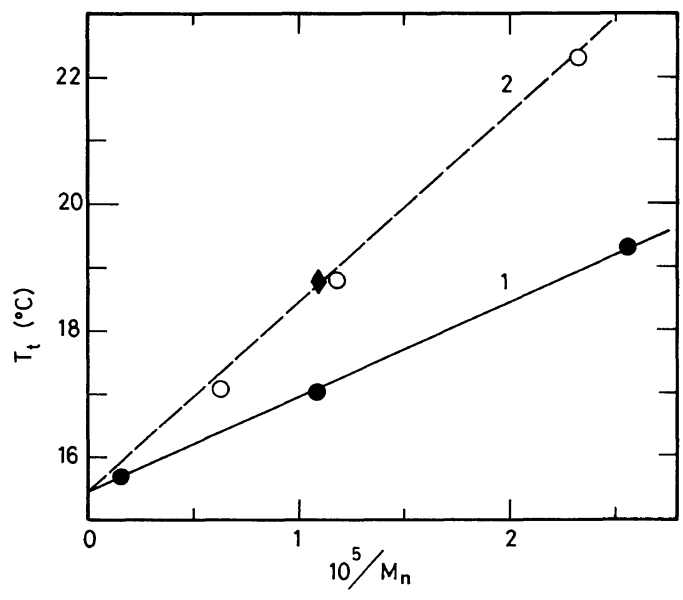

Figure 6. Helix-coil transition temperatures of EBE copolymer series (2) and of PELG homopolymer series (1) plotted against reciprocal number-average molecular weights $M_{n}^{-1}$. Designation $\downarrow$ denotes the result obtained from ultracentrifuge.

average molecular weights $M_{n}^{-1}$. The numberaverage molecular weights of EBE are those estimated from $P_{\mathrm{A}}$ given in Table I. For EBE-10, the value determined by the ultracentrifuge method is also included in Figure 6. The $M_{n}$ values of PELG homopolymers were estimated from the $[\eta]-M_{n}$ relationship for PMLG with almost the same molecular weight distribution as that of PELG, on the assumption that methyl and ethyl residues may be treated equally for solution viscosity. As is obvious from Figure 6, the experimental points for PELG 
homopolymers and those and for EBE copolymers fall on different straight lines. The slope of the straight line for the EBE copolymer series is twice that for the PELG homopolymer series. This fact supports the applicability of eq 5 and 6 ; in other words, EBE copolymers may be regarded as oncebroken rods.

\section{Molecular Conformations of EBE Block Copolymers in Solid Film}

The infrared spectra in the region of $4000-500$ $\mathrm{cm}^{-1}$ of solid films of EBE copolymers and PELG-2 homopolymer both cast from trifluoroethanol are shown in Figure 7. The spectrum of PELG is essentially the same as that of PMLG. ${ }^{22}$ The amide I, II, and V bands of these EBE block copolymers appear at 1650,1550 , and $620 \mathrm{~cm}^{-1}$, respectively, at just the same wave number for PELG-2. The wave number $620 \mathrm{~cm}^{-1}$ of the amide V band of PELG is somewhat shifted from $615 \mathrm{~cm}^{-1}$ for PMLG. A band associated with the $\mathrm{C}=\mathrm{C}$ torsion and the $\mathrm{CH}$ out-of-plane modes is observed at around $970 \mathrm{~cm}^{-1}$. The copolymer compositions estimated from the intensity of this band relative to that at $1120 \mathrm{~cm}^{-1}$ are in fairly good agreement with those obtained from the elemental analysis. These results imply that the E-block component in EBE block copolymers assumes the $\alpha$-helical conformation, and moreover, that the helix content of EBE block copolymers is nearly the same as that of PELG homopolymer.

Estimation of Micro-Heterophase Structure of EBE

Block Copolymers in Film by Means of an

Equivalent Mechanical Model for Dynamic

Viscoelastic Behavior

If the mutual solubility of component block chains of the block copolymers of A-B or A-B-A type is small, a well-defined microheterophase structure may be formed as a result of microphase separation.

Now we treat the dynamic mechanical spectra by an appropriate equivalent mechanical model. To begin with, we express the complex modulus $E^{\prime}$ of a two-phase mixture system composed of A and B polymer components using the parallel model indicated by eq 7 and the series model indicated by eq 8 ,

$$
E^{*}=\left(1-\phi_{\mathrm{B}}\right) E_{\mathrm{A}}^{*}+\phi_{\mathrm{B}} E_{\mathrm{B}}{ }^{*}
$$

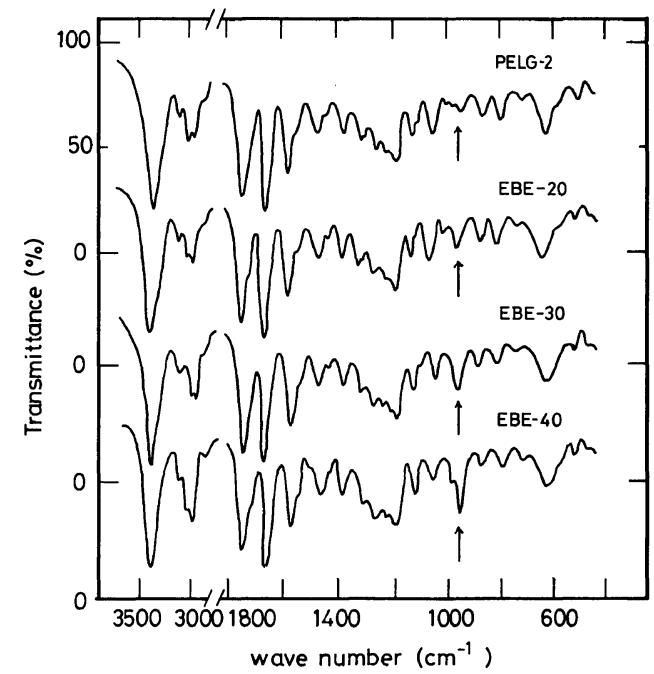

Figure 7. Infrared spectra of unoriented solid films of EBE copolymers and PELG homopolymer cast from trifluoroethanol.

$$
\frac{1}{E^{*}}=\frac{1-\phi_{\mathrm{B}}}{E_{\mathrm{A}}{ }^{*}}+\frac{\phi_{\mathrm{B}}}{E_{\mathrm{B}}{ }^{*}}
$$

where $E_{\mathrm{A}}{ }^{*}$ and $E_{\mathrm{B}}{ }^{*}$ are the complex moduli of $\mathrm{A}$ and $\mathrm{B}$ components, respectively, and $\phi_{\mathrm{B}}$ is the volume fraction of B component.

Takayanagi ${ }^{23}$ proposed, by combining eq 7 and 8 , an equivalent mechanical model composed of elements connected partly in series and partly in parallel to describe a two-phase mixture in which B component domains are distributed as the inclusion phase in the matrix of A component. This model gives $E^{*}$ for the complex modulus,

$$
\frac{1}{E^{*}}=\frac{\psi}{\lambda E_{\mathrm{B}}{ }^{*}+(1-\lambda) E_{\mathrm{A}}{ }^{*}}+\frac{1-\psi}{E_{\mathrm{A}}{ }^{*}}
$$

where $\lambda$ and $\psi$ are the parameters expressing the mixing state of the system, and the relative magnitude of $\lambda$ to $\psi$ can be interpreted as the ratio of the parallel to the series character. The product $\lambda \psi$ is equal to the volume fraction $\phi_{\mathrm{B}}$ of the inclusion phase. Further, if $E^{*}$ is separated into real and imaginary parts, expressions for the storage modulus $E^{\prime}$ and the loss modulus $E^{\prime \prime}$ are obtained as,

$$
\begin{aligned}
E^{\prime} & =\left|E^{*}\right| \cos \delta \\
E^{\prime \prime} & =\left|E^{*}\right| \sin \delta
\end{aligned}
$$

The micro-heterophase structure formed in our 
(a)
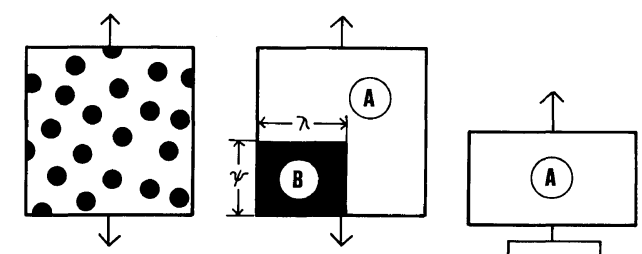

(b)
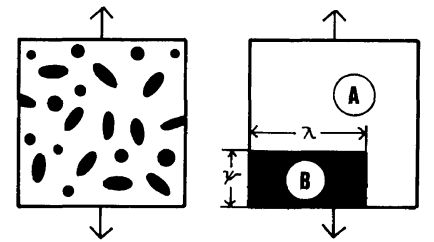

Figure 8. Schematic representation of equivalent mechanical models for two-phase mixture systems including (a) spherical and (b) cylindrical inclusion phases.

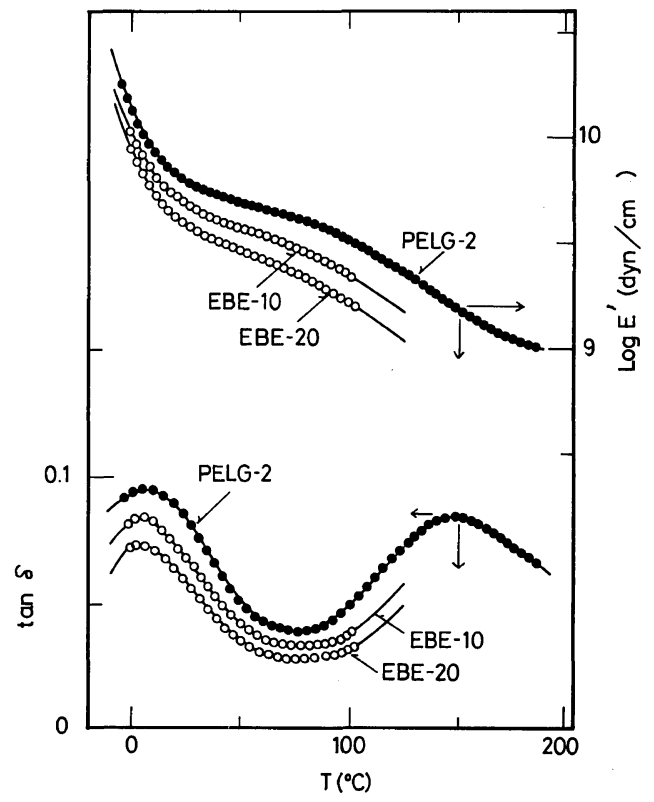

Figure 9. Temperature dependences of $\tan \delta$ and $\log$ $E^{\prime}$ for EBE-10, EBE-20, and PELG-2 membranes cast from trifluoroethanol.

EBE block copolymers can be treated by eq 9, since the E-block and B-block of EBE take the same conformations as those of PELG and polybutadiene homopolymers, respectively, and furthermore, the inclusion phase is rubber-like and its elastic Poisson ratio is equal to 0.5 . In Figure 8, the construction of the equivalent mechanical model given by eq 9 is schematically shown for systems with the inclusion phases of the same $\phi_{\mathrm{B}}$ but having different shapes. If

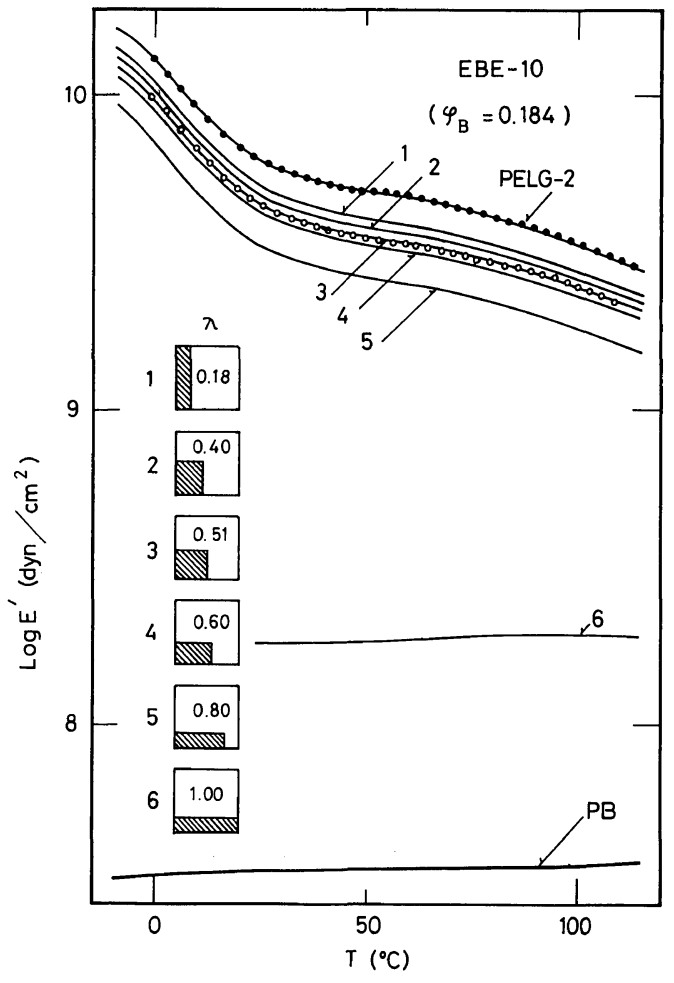

Figure 10. Temperature dependences of $\log E^{\prime}$ for EBE-10 membrane. Calculated curves $1-6$ are compared with experimental data.

the shape of the inclusion phase is spherical, the values of $\lambda$ and $\psi$ are given by, ${ }^{24}$

$$
\begin{aligned}
& \lambda=\left(2+3 \phi_{\mathbf{B}}\right) / 5 \\
& \psi=5 \phi_{\mathbf{B}} /\left(2+3 \phi_{\mathbf{B}}\right)
\end{aligned}
$$

However, if this shape deviates from a sphere, the contribution of $\lambda$ to $\phi_{\mathrm{B}}$ increases, and hence the model may approach series one shown in Figure 8b.

Figure 9 shows the temperature dependence of the loss tangent $\tan \delta$ and the storage modulus $E^{\prime}$ for PELG-2, EBE-10, and EBE-20 films of about $50 \mu \mathrm{m}$ thick cast from a 4:1 chloroformtrifluoroethanol mixture. The glass transition temperature $T_{\mathrm{g}}$ of polybutadiene is very low. ${ }^{25}$ For PELG, a sharp $\beta$-peak is observed in the loss tangent spectrum at about $0^{\circ} \mathrm{C}$, at which the storage modulus curve exhibits a large drop. Such behavior is attributed to the disordering of side chains of the polypeptide. The $\beta$-peak temperature for PELG obtained here is lower than that for PMLG, $15^{\circ} \mathrm{C}$. 
A-B-A Blockpolymers Containing Poly(ethyl glutamate)

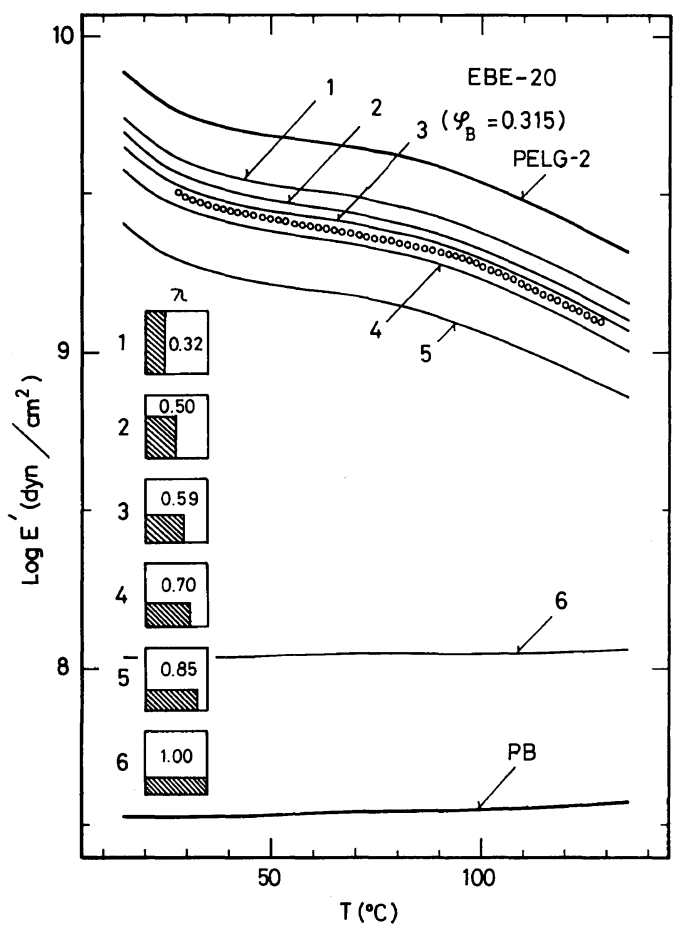

Figure 11. Temperature dependences of $\log E^{\prime}$ for EBE-20 membrane. Calculated curves $1-6$ are compared with experimental data.

The $\beta$-peaks of EBE copolymers appear at the same temperature as do those of PELG, but the height of $\tan \delta$ is lower than that for PELG. The $\alpha$-peak for PELG that may be assigned to the main chain structure is located at about $150^{\circ} \mathrm{C}$.

In Figures 10 and 11, the dynamic elastic modulus $E^{\prime}$ calculated by means of eq 9 and 10 is compared to the experimental data for EBE-10 and EBE-20, respectively. The data on polybutadiene was taken from the work by Keskkula. ${ }^{25}$ In Figure 10 , curves $1-6$ were obtained for different $\lambda$ values with $\phi_{\mathrm{B}}$ fixed at 0.184 . Curve 3 corresponds to the case of a spherical inclusion phase. The fact that the experimental points fall on curve 3 implies that the polybutadiene domains in EBE-10 block copolymer film are spherical. For EBE-20 (see Figure 11), however, the experimental points fall between curves 3 and 4 . This result indicates that the shape of microphase domains in EBE-20 is deviated from sphere and may be cylindrical microphase. These experimental results are summarized in Table III, in which $\lambda_{\mathrm{c}}$ denotes the value for spherical domain. It
Table III. Parameters $\phi_{\mathrm{B}}$ and $\lambda$ for EBE-10 and EBE-20

\begin{tabular}{ccccc}
\hline & \multicolumn{1}{c}{ Butadiene } & & & \\
\cline { 2 - 3 } & $\phi_{\text {Sample code }} \%$ & & $\lambda\left(\right.$ obs $\left.^{\mathrm{d}}\right)$ & $\lambda_{\mathrm{c}}$ \\
& $\mathrm{mol}$ & & & \\
\hline EBE-10 & 10.5 & 0.184 & 0.53 & 0.51 \\
EBE-20 & 19.4 & 0.315 & 0.63 & 0.59 \\
\hline
\end{tabular}

should be added that the $\phi_{\mathrm{B}}$ values used in these calculations were obtained from the copolymer composition by assuming that the chain conformation of polybutadiene is in the unperturbed state. ${ }^{6}$ The domain structures estimated here will be investigated further in a forthcoming paper from different points of view.

\section{REFERENCES}

1. Y. Yamashita, Y. Iwaya, and K. Ito, Macromol. Chem., 176, 1207 (1975).

2. B. Perly, A. Douy, and B. Gallot, Macromol. Chem., 177, 2569 (1976).

3. A. Douy and B. Gallot, Polym. Eng. Sci., 17, 523 (1977).

4. A. Nakajima, T. Hayashi, K. Kugo, and K. Shinoda, Macromolecules, 12, 840 (1979).

5. A. Nakajima, K. Kugo, and T. Hayashi, Macromolecules, 12, 844 (1979).

6. A. Nakajima, K. Kugo, and T. Hayashi, Polym. J., 11, 995 (1979).

7. T. Hayashi, Guan-Wen Chen, and A. Nakajima, Rep. Prog. Polym. Phys. Jpn., 23, (1980), submitted.

8. T. Hamada and A. Takizawa, J. Appl. Polym. Sci., 18, 1443 (1974).

9. Y. Nose, Cleveland Clinic, Cleveland, Ohio, USA, private communication.

10. Z. Provda, Collect Czech. Chem. Commun., 24, 2083 (1959).

11. S. Sugai, K. Kamashima, S. Makino, and J. Noguchi, J. Polym. Sci., A-2, 4, 183 (1966).

12. J. P. Greenstein and M. Winity, "Chemistry of the Amino Acids" Vol. 2, John Wiley \& Sons Inc. New York, N.Y., 1961, p 927.

13. F. H. C. Stewart and R. Ledger, Aust. J. Chem., 18, 1477 (1965).

14. T. Kotaka, N. Donkai, and H. Inagaki, J. Polym. Sci., A-2, 9, 1379 (1971).

15. D. A. Yphantis, Biochemistry, 3, 297 (1964).

16. S. Tanaka and A. Nakajima, Bull. Inst. Chem. Res., Kyoto Univ., 54, 229 (1976).

17. Y. Hashimoto, A. Aoyama, Y. Imanishi, and T. Higashimura, Biopolymers, 15, 2407 (1976). 


\section{G.-Wen Chen, T. Hayashi, and A. Nakajima}

18. A. Nakajima and T. Hayashi, Bull. Inst. Chem. Res., Kyoto Univ., 46, 62 (1968).

19. S. Lifson and A. Roig, J. Chem. Phys., 34, 1963 (1961).

20. K. Nagai, J. Chem. Phys., 34, 887 (1961).

21. A. Teramoto, T. Yamashita, and H. Fujita, J. Chem. Phys., 46, 1919 (1967).

22. T. Hayashi, K. Kugo, and A. Nakajima, Preprint, 24th Kobe Meeting of the Society of Polymer Science, Japan, Kobe, 1978, p 49.

23. M. Takayanagi, Mem. Fac. Eng. Kyushu Univ., 23, 1 (1963).

24. M. Takayanagi, S. Uemura, and S. Minami, J. Polym. Sci., C, No. 5, 113 (1965).

25. H. Keskkula and S. G. Turley, J. Polym. Sci., B, 7, 697 (1969). 